\title{
On the Capacity of Hybrid Wireless Networks with Opportunistic Routing
}

\author{
Tan Le, Yong Liu \\ Department of Electrical and Computer Engineering \\ Polytechnic Institute of New York University \\ Six MetroTech Center, Brooklyn, NY 11201 \\ tle04@students.poly.edu, yongliuapoly.edu
}

\begin{abstract}
This paper studies the capacity of hybrid wireless networks with opportunistic routing (OR). We first extend the opportunistic routing algorithm to exploit high speed data transmissions in infrastructure network through base stations. We then develop linear programming models to calculate the end-to-end throughput bounds from multiple source nodes to single, as well as, multiple destination nodes. The developed models are applied to study several hybrid wireless network examples. Through case studies, we investigate several factors that have significant impacts on the hybrid wireless network capacity under opportunistic routing, such as node transmission range, density and distribution pattern of base stations (BTs), number of wireless channels on wireless nodes and base stations, etc. Our numerical results demonstrate that opportunistic routing could achieve much higher throughput on both ad-hoc and hybrid networks than traditional unicast routing (UR). Moreover, opportunistic routing can efficiently utilize base stations and achieve significantly higher throughput gains in hybrid wireless networks than in pure ad-hoc networks especially with multiple-channel base stations.
\end{abstract}

\section{Introduction}

New portable devices, such as iPhone, PDAs are increasingly equipped with strong communication and computation capabilities. They can host a wide range of applications, such as web browsing, audio/video streaming, online gaming, etc. Most devices have multiple radio interfaces and support different wireless protocols, such as Bluetooth, $\mathrm{Wi}-\mathrm{Fi}$, and 3G. It has become critical for such devices to efficiently utilize resources available in a hybrid wireless networking environment to achieve high data throughput and support bandwidth-intensive applications.

Recently, Opportunistic Routing (OR) was proposed to improve the throughput for ad-hoc networks. In this paper, we explore the gain of integrating OR with hybrid wireless networks that consist of ad-hoc wireless nodes and base stations connected to a wireline infrastructure. We first extend the opportunistic routing algorithm to exploit high speed data transmissions in infrastructure network through base stations. We then develop linear programming models to calculate the end-to-end throughput bounds from multiple source nodes to single, as well as, multiple destination nodes. The developed models are applied to study several hybrid wireless network examples. Through case studies, we investigate several factors that have significant impacts on the hybrid wireless network capacity under OR, such as density and distribution pattern of Base Stations, number of wireless channels on wireless nodes and BTs, etc.

The contribution of this paper is four-fold:

1. We propose a simple method to extend OR to hybrid wireless networks. We develop new transmission cost metrics and forwarding priority rules to take into account candidate routes through BTs and infrastructure network.

2. We develop linear programming models to calculate end-to-end throughput bounds from multiple source nodes to single, as well as, multiple destination nodes.

3. We demonstrate through case studies that OR can efficiently utilize BTs and achieve significantly higher throughput gains in hybrid wireless networks than in pure ad-hoc networks. And the throughput gain of OR is also higher than that of UR in hybrid networks.

4. We systematically evaluate several factors determining the throughput gains of OR in hybrid wireless networks.

The rest of the paper is organized as follows. We briefly review the related works in Section 2. In Section 3, we present the extension of OR to hybrid wireless networks and the LP models to characterize the throughput bounds from multiple sources to single destination, and from multiple sources to multiple destinations. Case studies on several example hybrid wireless networks are presented in Section 4. The paper is concluded in Section 5. 


\section{Background and Related Work}

The throughput bound and capacity of ad-hoc and hybrid wireless networks have been studied extensively in the past. Well-known papers [13] and [14] developed analytical methods to calculate the capacity of mobile and ad-hoc networks. The works in [2][3][4][5][6][7] investigated the capacity of ad-hoc networks with infrastructure support in different cases and scenarios under UR. Recently, the topic Opportunistic Routing on ad-hoc networks attracted lots of interest [8][9][10][11][12]. In [11], the authors studied the opportunistic routing protocol ExOR, which dynamically chooses paths on a per-transmission basis in a wireless network to efficiently improve the throughput. To illustrate the idea of $\mathrm{OR}$, in Figure 1, $A$ wants to send packets to $D . B_{1}, B_{2}$ and $B_{3}$ are closer to $D$ and are chosen as the candidate forwarders. After one broadcast from $A, B_{1}, B_{2}$, and $B_{3}$ all receive the packet. Assuming $B_{1}$ has the highest forwarding priority, so it will take over and broadcast the packet to its candidate forwarders $C_{1}, C_{2}$ and $C_{3}$. Assume highest priority node $C_{1}$ misses the packet, so $C_{2}$ will take over and forward the packet to its destination $D$. In [8], by integrating opportunistic routing with network coding, a new protocol MORE leads to significant throughput improvement in both unicast and multicast cases. In [9], the authors introduced the robust distribution opportunistic routing scheme base on ETX metric that can find the optimal path from source to destination. Authors of [12] conducted a systematic performance evaluation, taking into account node densities, channel qualities and traffic rates to identify the cases when opportunistic routing makes sense. The recent work from K.Zeng et al. [1] proposed the method to calculate the maximum throughput between two end nodes with Opportunistic Routing in ad-hoc networks. The main focus of this paper is to study the throughput improvement of OR in hybrid wireless networks.

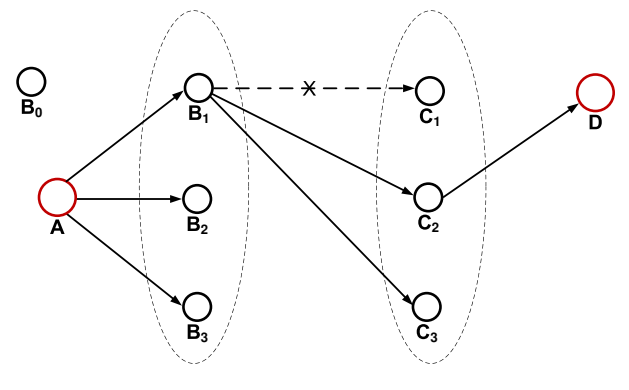

Fig. 1. Opportunistic Routing on ad-hoc network

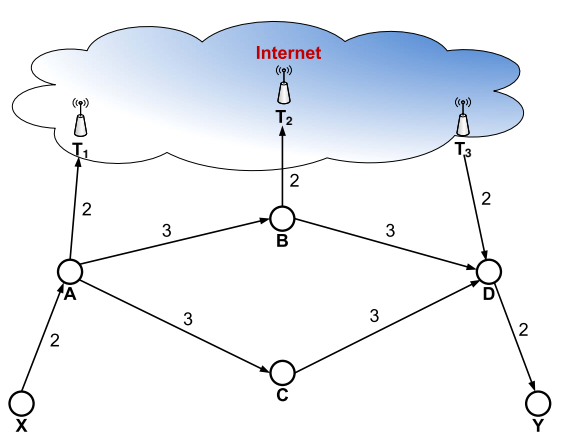

Fig. 2. Simple example of hybrid wireless network

\section{Capacity of Hybrid Wireless Networks with OR}

\subsection{Network Model}

We consider a hybrid wireless network consisting of wireless nodes and Wi-Fi Base Stations (BTs). Wireless nodes are equipped with radio interfaces and can communicate with each other through multi-hop ad-hoc transmissions. BTs are connected to the Internet using high bandwidth wireline connections. If a wireless node is within the coverage of a BT, it can communicate with the BT using single-hop infrastructure mode. Optionally, a wireless node might have a connection to a $3 \mathrm{G}$ base station that covers all wireless nodes under consideration. The optional $3 \mathrm{G}$ connection can be used as a control channel for nodes to exchange control information, such as the geographical locations of nodes. Packets can be transmitted using two transmission modes: ad-hoc mode and infrastructure mode. We assume all nodes in the network are cooperative and forward each other's packets to their destinations with Opportunistic Routing. Here are some assumptions on hybrid wireless networks under study:

- There are $N_{1}$ static wireless nodes randomly located in a square area. There are $N_{2}$ Wi-Fi Base Stations in the same area.

- Wireless nodes are homogeneous. They have the same set of transmission rates, and equivalent effective transmission ranges. 
- Assume the coverage areas of BTs do not overlap with each other. Each wireless node could connect to at most one BT.

- Source node transmits data with OR through relay nodes to destination. If the relay node is a wireless node, it uses OR to forward the packet to the next-hop node (relay or the final destination). If a relay node is a BT, it forwards the packet to the next-hop node through direct single-hop transmission.

- Through a separate control channel (e.g., 3G), every node knows the geographical locations of its neighbors, base stations and the destination node. Nodes then could differentiate the transmissions in wireless domain and wireline domain when making route selection over hybrid wireless network.

- We study two different models for data transmissions in hybrid wireless networks:

1. Single-channel model: In this model, all BT nodes and wireless nodes are equipped with a single radio interface. They use the same frequency spectrum to communicate with each other. In other words, infrastructure and ad-hoc transmissions share the same wireless channel. Wireless nodes use OR and BTs use UR to forward packets toward their destinations. Since every BT node only has a single wireless channel, it could communicate with no more than one wireless node at any given time.

2. Multiple-channel model: In this model, infrastructure and ad-hoc transmissions operate at non-overlapping frequency ranges. Wireless nodes in the coverage of a BT can simultaneously communicate with the BT using infrastructure mode and other wireless nodes using ad-hoc mode. Moreover, every BT node has multiple wireless channels, so it can communicate with multiple wireless nodes simultaneously. Wireless nodes use OR and BTs use UR to forward packets. If the Candidate Relay Set (CRS) of a wireless node consists of a BT and some wireless nodes, the wireless node simultaneously employs infrastructure and ad-hoc transmissions to push the same packet to the BT and wireless nodes respectively.

\subsection{Concurrent Transmitter Sets}

The biggest challenge of studying the capacity of wireless networks is to model the conflicts between wireless links. The concept of Concurrent Transmitter Sets (CTS) was proposed in [1] to calculate the end-to-end throughput in ad-hoc networks with OR. We extend the CTS concept to study the capacity of hybrid wireless networks.

With OR, a transmitter has multiple forwarding candidates in its Candidate Relay Set (CRS). Let all links from a transmitter to nodes in its CRS as links associated with that transmitter. In a hybrid wireless network, Conservative CTS (CCTS) is a set of transmitters (including the BTs) that when all of them are transmitting simultaneously, all links associated with them are still usable (no interfere with any other link [1]). However, such a requirement is too restrictive. Data from a transmitter can be forwarded to the next hop as long as one forwarding candidate in its CRS receives the data. To account for this, Greedy CTS (GCTS) is a set of transmitters (including the BTs) that when all of them are transmitting data simultaneously, at least one link associated with each transmitter is usable. This leads to the maximum end-to-end throughput. A maximal CCTS (GCTS) is a CCTS (GCTS) that is not a true subset of any another CCTS (GCTS).

For the single-channel model, infrastructure transmissions could interfere with ad-hoc transmissions. A BT cannot send and receive data with more than one wireless node at a particular time. For the multiple-channel model, BTs can send and receive data with multiple nodes simultaneously in infrastructure mode. Infrastructure transmissions have no conflict with ad-hoc transmissions. Due to the assumed non-overlapped BT coverage areas, infrastructure transmissions of different BTs are also conflict-free. Data transmissions between BTs are in the wireline domain and will not interfere with any wireless transmissions. Consequently, directed links between BTs, and directed links between a BT and its associated end nodes can be activated at anytime without introducing interference to any other link in the network. With the assumption of the number of wireless channels on each BT is big enough that it could sending and receiving data with all associated nodes simultaneously, all BT nodes can be included in all CTSs. An example of CTS is illustrated in Figure 2. A link $i j$ in the graph indicates node $j \in$ CRS of node $i$ and they are in the transmission range of each other. Assume source node $A$ needs to send data to destination node $D$ with the relays $B, C$, base stations $T_{1}, T_{2}$ and $T_{3}$. We will find the CTSs for the two different models.

1. Single-channel model: Pairs of nodes $(A, B),(B, C),(C, A)$ could not be included in the same CCTS. The reason is that two sets of links associated with each pair of nodes are not interference free. Also the pairs of of nodes $\left(B, T_{3}\right)$ and $\left(C, T_{3}\right)$ could not be included in the same CCTS because their links to node $D$ are not interference free. So the maximal Conservative CTSs in this case are: $\left\{A, T_{1}, T_{2}, T_{3}\right\},\left\{B, T_{1}, T_{2}\right\},\left\{C, T_{1}, T_{2}\right\}$. The maximal Greedy CTSs in this case are exactly the same as the above maximal CCTSs. When all nodes 
in each of these GCTSs transmitting simultaneously, usable links associated with each node are: $\mathbf{A}: A T_{1}$; $\mathbf{B}: B T_{2} ; \mathbf{C}: C D ; \mathbf{T}_{\mathbf{1}}: T_{1} T_{3} ; \mathbf{T}_{\mathbf{2}}: T_{2} T_{3}, \mathbf{T}_{\mathbf{3}}: T_{3} D$.

2. Multi-channels model: For Conservative CTSs, pairs of nodes $(A, B),(B, C),(C, A)$ could not be included in the same CCTS. So the maximal CCTSs in this case are: $\left\{A, T_{1}, T_{2}, T_{3}\right\},\left\{B, T_{1}, T_{2}, T_{3}\right\},\left\{C, T_{1}, T_{2}, T_{3}\right\}$. On the other hand, for GCTSs, there are only pairs of nodes $(A, C)$ and $(B, C)$ could not be included in the same GCTS. It is because the only link $C D$ associated with node $C$ will be not usable whenever nodes $A$ or $B$ activated to transmit data. So the maximal Greedy CTSs in this case are: $\left\{A, B, T_{1}, T_{2}, T_{3}\right\},\left\{C, T_{1}, T_{2}, T_{3}\right\}$. When all nodes in each of these GCTSs transmitting simultaneously, usable links associated with each node are: $\mathbf{A}: A T_{1} ; \mathbf{B}: B T_{2} ; \mathbf{C}: C D ; \mathbf{T}_{\mathbf{1}}: T_{1} T_{3} ; \mathbf{T}_{\mathbf{2}}: T_{2} T_{3}, \mathbf{T}_{\mathbf{3}}: T_{3} D$.

\subsection{Opportunistic Routing Model}

In OR, a transmitter selects neighbors "closer", i.e., with lower transmission cost, to the destination as candidate forwarders in CRS. Forwarders in CRS are ranked based on their "closeness" to the destination. Since there is no preset route to a destination with $\mathrm{OR}$, it is impossible to determine the accurate transmission cost from a node to a destination. In a pure ad-hoc network, one can use the geographic distance between a node $i$ and destination node $j$ to measure the packet transmission cost from $i$ to $j$ through ad-hoc network. For hybrid wireless networks, we propose a new metric that takes into account the low transmission cost through the infrastructure network. We assume costs of the infrastructure transmissions between BTs are negligible. Then the cheapest transmission from $i$ to $j$ through infrastructure network is for $i$ to transmit a packet destined to $j$ first to its closest BT, $T_{i}$. Then $T_{i}$ transmits the packet to a BT $T_{j}$ that is the closest to node $j$. Finally, $T_{j}$ sends the packet to $j$. If $i$ is directly covered by $T_{i}$, we use geographic distance $d_{i T_{i}}$ between $i$ and $T_{i}$ to estimate the transmission cost $\hat{d}_{i T_{i}}$ from $i$ to $T_{i}$. If $i$ is not in the coverage of $T_{i}$, we choose a node $k_{i}$ in $T_{i}$ 's coverage that is the closest to $i$ as a relay node. All packets from $i$ to $T_{i}$ will be first sent to $k_{i}$ using the ad-hoc mode, then be relayed to $T_{i}$ using the infrastructure mode. Consequently, the transmission cost is estimated as $\hat{d}_{i T_{i}}=d_{i k_{i}}+d_{k_{i} T_{i}}$. Similarly, the transmission cost from $T_{j}$ to $j$ can be estimated as $\hat{d}_{T_{j} j}$. The total transmission cost through the infrastructure network is then estimated as $\hat{d}_{i j}=\hat{d}_{i T_{i}}+\hat{d}_{T_{j} j}$. The effective transmission cost $\bar{d}_{i j}$ from $i$ to $j$ in the hybrid wireless network is the minimum of the cost of pure ad-hoc transmission and that of transmission through infrastructure:

$$
\bar{d}_{i j}=\min \left(\hat{d}_{i j}, d_{i j}\right)
$$

For the example in Figure 2, the source node is $X$ and the destination node is $Y$. Assuming the geographic distances are $d_{X Y}=9, d_{X B}=5, d_{X D}=7, d_{A Y}=5, d_{B Y}=7$. The transmission cost using infrastructure network can be estimated as:

$$
\hat{d}_{X Y}=\left(d_{X A}+d_{A T_{1}}\right)+\left(d_{T_{3} D}+d_{D Y}\right)=(2+2)+(2+2)=8
$$

So $\bar{d}_{X Y}=\min \left(\hat{d}_{X Y}, d_{X Y}\right)=8$.

In OR, a forwarding candidate is utilized to transmit a packet if and only if it receives the packet and all other candidates with higher priority in the CRS don't receive the packet. To study the capacity of OR, we need to calculate the effective forwarding rate of a link between a transmitter $i$ to each of its forwarding candidate $k$. Let $i$ sends data to its forwarding candidate set with rate $R . J(i)$ be the candidate forwarding set for $i$, and $J(i)=\left\{i_{1}, i_{2}, \ldots, i_{r}\right\}$. The priority order to forward packets from $i$ is $i_{1}<i_{2}<\ldots<i_{q}<\ldots<i_{r}, 1 \leqslant q \leqslant r$. Let $p_{i k}$ be the Packet Reception Ratio (PRR) between $i$ and $k . p_{i k}$ theoretically depends on distance between $i$ and $k$, end node density around the position of nodes $i$ and $k$, and the MAC scheduling scheme. Then the effective forwarding rate $\tilde{R}_{i i_{q}}$ on link $\left\langle i i_{q}\right\rangle$ is:

$$
\tilde{R}_{i i_{q}}=R p_{i i_{q}} \prod_{k=0}^{q-1}\left(1-p_{i i_{k}}\right)
$$

\subsection{Throughput Bound to Single Destination}

Given basic models studied in previous sections, we now proceed to study the capacity of hybrid wireless networks with OR. We start with the case that multiple sources send data to the same destination. As summarized in Table 1, 


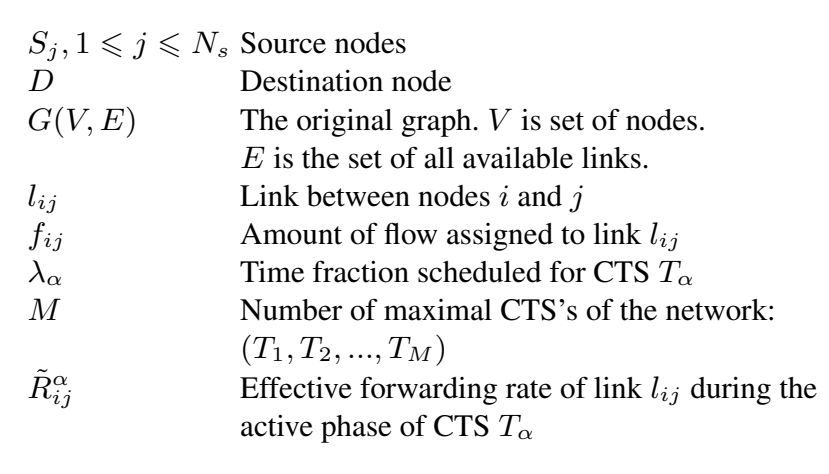

Table 1. Notation on Linear Programming Formulations

there is a set $\mathcal{S}$ including $N_{s}$ source nodes $\left\{S_{1}, \cdots, S_{N_{s}}\right\}$ sending traffic to the same destination node $D$. From the original network, we create a connected graph $G=(V, E) . V$ is the set of nodes, including end nodes and BT nodes. $E$ is the set of all available links, including ad-hoc links and infrastructure links. Let $f_{i j}$ be the amount of traffic sent on link $l_{i j}$. We are interested in finding out the bound of end-to-end throughput from source nodes in $\mathcal{S}$ to $D$.

Assuming there are $M$ maximal CTS's $\left(T_{1}, T_{2}, \ldots, T_{M}\right)$. At any time, when a CTS is scheduled to transmit, nodes in the scheduled CTS could transmit packets simultaneously. Let $\lambda_{\alpha}$ be the time fraction that CTS $T_{\alpha}$ is scheduled. We need to calculate the effective forwarding rate $\tilde{R}_{i j}^{\alpha}$ for each link $\langle i, j\rangle$ under each CTS $T_{\alpha}$. If a CCTS $T_{\alpha}$ is scheduled and $i \in T_{\alpha}$, all links associated with $i$ are usable, therefore $\tilde{R}_{i j}^{\alpha}=\tilde{R}_{i j}, \forall j \in J(i)$, which is calculated in (3); if $i \notin T_{\alpha}, \tilde{R}_{i j}^{\alpha}=0$. If a GCTS $T_{\alpha}$ is scheduled and node $i \in T_{\alpha}$, some links associated with $i$ maybe not usable. Let $\psi_{i j}^{\alpha}$ be a binary variable for the usability of link $\langle i, j\rangle$ under a GCTS $T_{\alpha}$, then we have $\tilde{R}_{i j}^{\alpha}=\psi_{i j}^{\alpha} \tilde{R}_{i j}, \forall j \in J(i)$.

Let $H_{S_{j}}$ be the sending rate from source $S_{j}$ toward the destination $D$. We have the following LP optimization formulation to characterize the throughput bound with single destination.

$$
\max \sum_{j=1}^{N_{s}} H_{S_{j}}
$$

subject to,

$$
\begin{gathered}
f_{i j} \geqslant 0, \text { and } \quad f_{i j}=0, \text { if } \quad j \notin J(i), \forall\langle i, j\rangle \in E, \\
\sum_{\langle i, j\rangle \in E} f_{i j}=\sum_{\langle j, i\rangle \in E} f_{j i}, \forall i \in V-\mathcal{S}-\{D\} \\
\sum_{\left\langle S_{j}, k\right\rangle \in E} f_{S_{j} k}-\sum_{\left\langle i, S_{j}\right\rangle \in E} f_{i S_{j}}=H_{S_{j}}, \forall S_{j} \in \mathcal{S} \\
\sum_{\langle D, i\rangle \in E} f_{D i}=0 \\
f_{i j} \leqslant \sum_{\alpha=1}^{M} \lambda_{\alpha} \tilde{R}_{i j}^{\alpha}, \quad \sum_{\alpha=1}^{M} \lambda_{\alpha} \leqslant 1 ; \lambda_{\alpha} \geqslant 0,1 \leqslant \alpha \leqslant M
\end{gathered}
$$

Equation (4) is to find the maximum amount of traffic sent out from all the source nodes $\left\{H_{S_{j}}\right\}$ to the destination. Constraint (5) specifies that the traffic on all links are none negative and there are no traffic from one node to its neighbor nodes that are not in its forwarding candidate set. Constraint (6) specifies flow conservation on all relay nodes. Constraint (7) specifies the flow conservations on all source nodes $S_{j}, 1 \leqslant j \leqslant N_{s}$. Constraint (8) states that no outgoing traffic from destination node $D$. Constraint (9) preserves that only one CTS could be activated to transmit at any given time and the traffic assigned on each link is no more than the aggregate effective forwarding rate of 
that link during all active phases of CTSs. Depending on what types of CTSs we used as the input of the above formulation, we will get different bounds. Conservative CTS (CCTS) leads to conservative upper bound, Greedy CTS (GCTS) leads to optimistic upper bound of the end-to-end throughput.

\subsection{Throughput Bound to Multiple Destinations}

Based on the formulation for the single destination, we develop a model to calculate the throughput bound from multiple sources to multiple destinations in hybrid wireless networks. Suppose there are a set $\mathcal{S}$ including $N_{s}$ source nodes $\left\{S_{i}, 1 \leqslant i \leqslant N_{s}\right\}$, and a set $\mathcal{D}$ including $N_{d}$ destination nodes $\left\{D_{j}, 1 \leqslant j \leqslant N_{d}\right\}$. In OR, at each node $i$, there are different candidate forward sets for different destinations. Let $J^{d}(i)$ be the candidate forwarding set for destination node $d$ at node $i, J^{d}(i)=\left\{i_{1}^{d}, i_{2}^{d}, \cdots\right\}$ with the priority order $\left\{i_{1}^{d}<i_{2}^{d}<\cdots\right\}$. Similar to (3), for the $q$-th forward candidate $i_{q}^{d}$ in $J^{d}(i)$, we can calculate the effective forwarding rate for destination $d$ on link $\left\langle i, i_{q}^{d}\right\rangle$ as

$$
\tilde{R}_{i i_{q}^{d}}^{(d)}=R p_{i i_{q}^{d}} \prod_{k=0}^{q-1}\left(1-p_{i i_{k}^{d}}\right), \quad 1 \leqslant q \leqslant\left|J^{d}(i)\right| .
$$

Since CTS is also defined based on forwarding sets for all nodes, we need to include destination information into the definition of CTS. More specifically, a Conservative CTS (CCTS) $T_{\alpha}$ is a set of transmitter-destination pairs $T_{\alpha}=\{(i, d(i)), i \in V, d(i) \in V\}$, such that all links $\left\{\langle i, j\rangle, \forall j \in J^{d(i)}(i), \forall(i, d(i)) \in T_{\alpha}\right\}$ are usable when all transmitters in CCTS are active. Similarly, a Greedy CTS (GCTS) $T_{\alpha}$ is a set of transmitter-destination pairs $T_{\alpha}=\{(i, d(i)), i \in V, d(i) \in V\}$ such that for each transmitter $i$ in GCTS, there exists at least one link $\langle i, j\rangle$, $j \in J^{d}(i)$, that is usable when other transmitters in GCTS are active.

Similar to the single destination case, we need to calculate the effective forwarding rate $\tilde{R}_{i j}^{\alpha(d)}$ on each link $\langle i, j\rangle$ for destination $d$ under each CTS $T_{\alpha}$. If a CCTS $T_{\alpha}$ is scheduled and $(i, d) \in T_{\alpha}$, all links from $i$ to nodes in $J^{d}(i)$ are usable, therefore $\tilde{R}_{i j}^{\alpha(d)}=\tilde{R}_{i j}^{(d)}, \forall j \in J^{d}(i)$, which is calculated in (10); if $(i, d) \notin T_{\alpha}, \tilde{R}_{i j}^{\alpha(d)}=0$. If a GCTS $T_{\alpha}$ is scheduled and $(i, d) \in T_{\alpha}$, some links associated with $i$ maybe not usable. Let $\psi_{i j}^{\alpha}$ be a binary variable for the usability of link $\langle i, j\rangle$ under a GCTS $T_{\alpha}$, then we have $\tilde{R}_{i j}^{\alpha(d)}=\psi_{i j}^{\alpha} \tilde{R}_{i j}^{(d)}, \forall j \in J^{d}(i)$.

Let $H\left(S_{i}, D_{j}\right)$ be the sending rate from source $i$ to the destination $j$, and $f_{i j}^{(d)}$ be the traffic on link $\langle i, j\rangle$ destined to $d$. We have the following LP optimization formulation to characterize the aggregate throughput bound.

$$
\max \sum_{i=1}^{N_{s}} \sum_{j=1}^{N_{d}} H\left(S_{i}, D_{j}\right)
$$

subject to,

$$
\begin{gathered}
f_{i j}^{(d)} \geqslant 0, \quad f_{i j}^{(d)}=0, \text { if } \quad j \notin J^{d}(i), \forall\langle i, j\rangle \in E, \forall d \in \mathcal{D} \\
\sum_{\langle i, j\rangle \in E} f_{i j}^{(d)}=\sum_{\langle j, i\rangle \in E} f_{j i}^{(d)}, \forall i \in V-\mathcal{S}-\{d\}, \forall d \in \mathcal{D} \\
\sum_{\left\langle S_{j}, k\right\rangle \in E} f_{S_{j} k}^{(d)}-\sum_{\left\langle i, S_{j}\right\rangle \in E} f_{i S_{j}}^{(d)}=H\left(S_{j}, d\right), \forall S_{j} \in \mathcal{S}, \forall d \in \mathcal{D} \\
\sum_{\langle d, i\rangle \in E} f_{d i}^{(d)}=0, \forall d \in \mathcal{D} \\
f_{i j}^{(d)} \leqslant \sum_{\alpha=1}^{M} \lambda_{\alpha} \tilde{R}_{i j}^{\alpha(d)}, \forall d \in \mathcal{D}, \sum_{\alpha=1}^{M} \lambda_{\alpha} \leqslant 1 ; \lambda_{\alpha} \geqslant 0,1 \leqslant \alpha \leqslant M
\end{gathered}
$$

Similar to the single destination case, constraints (12), (13), (14) and (15) specifies legitimate per-destination traffic flow on all links, relay nodes, source nodes, and destinations. Constraint (16) preserves that one CTS can be activated to transmit at any time, for each destination, the traffic assigned on each link is no more than total amount of traffic that could be delivered on that link during all active phases of CTSs. 


\section{Performance Evaluation}

In this section, we apply models developed in the previous section to study the throughput bound and capacity of hybrid wireless networks with OR in three different cases: Single Source to Single Destination, Multiple Sources to Single Destination and Multiple Sources to Multiple Destinations.

\subsection{Methodology}

We setup the case studies with different network sizes and different characteristics of the network in order to get the most accurate conclusions about the hybrid wireless network capacity. Based on the transmission range of transmitters, we developed a C++ program to calculate CTSs. Given node locations, the program calculates CTSs for both single-channel and multiple-channel models. The proposed LP method could be used for any type of packet loss model. For demonstration, we use a simple packet loss model on link $\langle i, j\rangle: p_{i j}=1-d_{i j} / L$, where $d_{i j}$ is the distance between $i$ and $j, L$ is the maximum transmission range. The node transmission rate is fixed at 10 packets/timeslot. We then calculate the effective forwarding rate on each link $\tilde{R}_{i j}^{\alpha}$ for each CTS. Then we use AMPL - CPLEX to solve the LP Problem to find the maximum throughput in each case. For each case study, we conduct multiple simulation runs and report the average of all runs.

To understand the gain of OR in hybrid wireless networks, we also compare the performance of OR with that of hybrid unicast routing in the same network setting. To calculate the throughput bound of UR, we first build up the link conflict graph out of the original graph. In the conflict graph, each vertex corresponds to a link in the original graph. There is a link between two vertexes in the conflict graph if two corresponding links in the original graph interfere with each other. By finding all maximal independent sets of vertexes in the conflict graph, we can find the maximal sets of links in the original graph that can be activated at the same time. Assuming there are $M$ maximal independent sets $\left(T_{1}, T_{2}, \ldots, T_{M}\right)$. At any time, one set can be scheduled to transmit and all links in the scheduled set can transmit simultaneously. Let $\lambda_{\alpha}$ be the time fraction that $T_{\alpha}$ is scheduled. The forwarding rate on link $\left\langle i i_{q}\right\rangle$ is:

$$
\tilde{R}_{i i_{q}}=R p_{i i_{q}}
$$

Then, we can reuse the LP formulation from (4) to (9) and from (11) to (16) to calculate the capacity of hybrid wireless networks under either OR or UR routing method.

\subsection{Single Source to Single Destination}

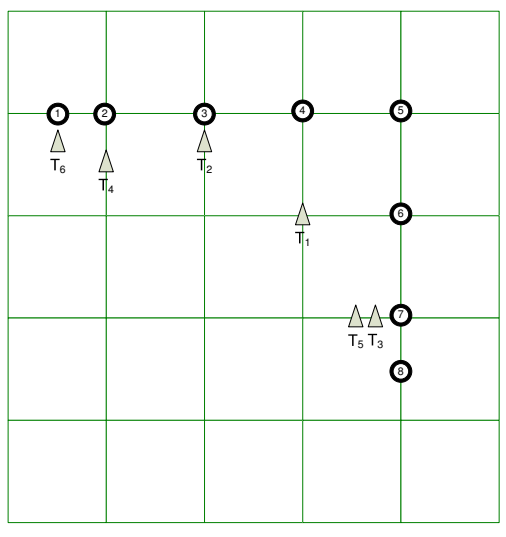

(a) Topology

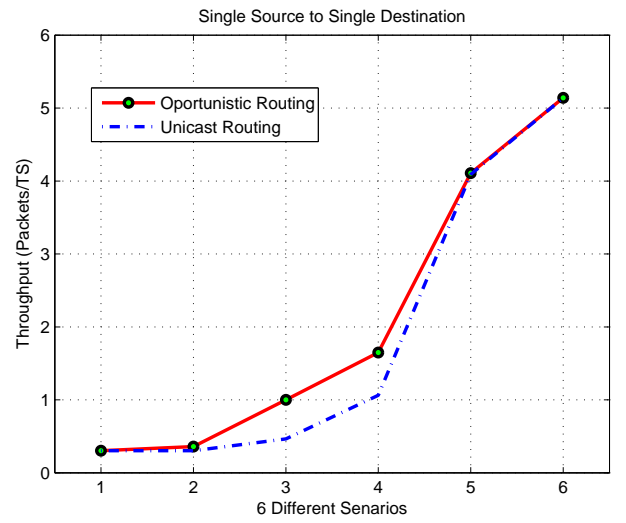

(b) Throughput

Fig. 3. End-to-End throughput improvement from single source to single destination

At first, we run the case studies with a small network setting. The network area is $500 \mathrm{~m} \times 500 \mathrm{~m}$. There are 8 wireless ad-hoc nodes. Nodes are located at the special positions as in Figure 3(a). Node 1 is the source, node 8 is the destination. The initial radio range of nodes is $110 \mathrm{~m}$. Source node and relay nodes send out packets with rate 
10 packets/timeslot. We start the experiment with pure ad-hoc transmissions. Then BTs are added with different parameters and positions. From this setup, we calculate the maximum end to end throughput at 6 different cases of BT locations. Case 1: no BT; Case 2: one BT in position $T_{1}$; Case 3: two BTs in positions $T_{2}$ and $T_{3}$; Case 4: two BTs in positions $T_{2}$ and $T_{3}$ with the radio range of every node from now on increased to $120 \mathrm{~m}$; Case 5: two BTs in position $T_{4}$ and $T_{5}$. Case 6: two BTs in position $T_{6}$ and $T_{3}$. We make the comparison between the OR and UR on the same network setting. The LP results of the bound of end to end throughput are showed on the Figure 3(b). First, we analyze throughput bound with OR. In ad-hoc mode, the bound of throughput from node 1 to node 8 is 0.3 packets/TS. All traffic routed through the path $1 \rightarrow 2 \rightarrow 3 \rightarrow \ldots \rightarrow 8$. The bottom necks on the maximum throughput path are the links: $2 \rightarrow 3,3 \rightarrow 4,4 \rightarrow 5,5 \rightarrow 6,6 \rightarrow 7$. In case 2 , when one BT is added to the network, throughput bound start gaining to 0.36 packets/TS since some additional traffic could be routed through infrastructure network over links $4 \rightarrow T_{1} \rightarrow 6$. Case 3 , when two BTs are located in the positions of $T_{2}$ and $T_{3}$, throughput bound increased to 1.0 packets/TS with more traffic could be routed through infrastructure network to get over "bottleneck" area. In case 4 , when the radio range of each node is slightly increased, the throughput bound is increased to 1.65 packets/TS since the packet loss ratios on links are reduced. Consequently the effective forwarding rates on wireless links are increased. When the positions of two BTs are changed to positions $T_{4}$ and $T_{5}$ in case 5 , the throughput is increased to 4.1 packets/TS. This is because all traffic is routed through high bandwidth infrastructure network through link $T_{4} \rightarrow T_{5}$. In case 6 , two BTs are located in position $T_{6}$ and $T_{3}$, closer to the source and destination nodes. That helps to improve the bound of throughput from node 1 to node 8 to 5.14 packets/TS. All traffic is routed through the infrastructure network from the source to the destination in a single path.

When wireless nodes use UR to forward data, throughput gets through a single path from the source node to the destination node. The throughput bound on each case is: Case 1: 0.3 packets/TS; Case 2: 0.3 packets/TS; Case 3: 0.47 packets/TS; Case 4: 1.06 packets/TS; Case 5: 4.09 packets/TS; Case 6: 5.14 packets/TS. Throughput bound of the network with OR will be higher than with UR when the optimal solution using more than one path to forward data toward the destination node. There for in cases 2, 3, 4 and 5, throughput gain with OR are higher than that with UR. But both routing methods get the same throughput bound for the cases 1 and 6 . From the above results, we can see that infrastructure network could significantly increase the end-to-end throughput of ad-hoc network with OR. The numbers and locations of BTs are important and could significantly impact the end-to-end throughput. OR will outperform UR for the cases of using multi paths to get to the destination.

\subsection{Multiple Sources to Single Destination}

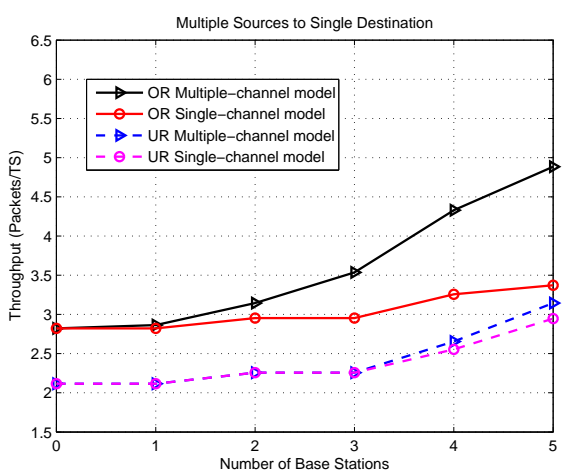

Fig. 4. Throughput bound on Random distribution of BTs with two different models

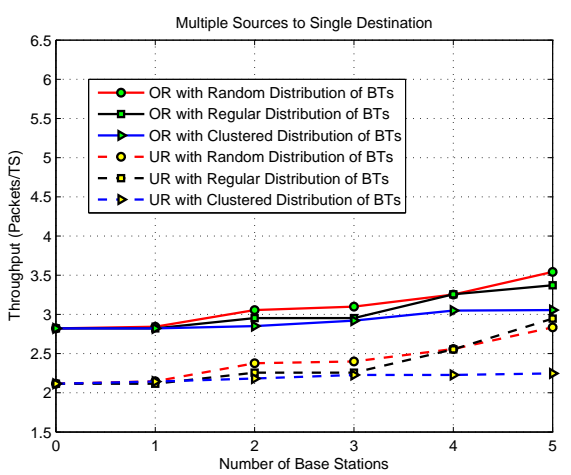

Fig. 5. Throughput bound on different distribution patterns of single-channel BTs

For the case of multiple sources and single destination, we studied two different settings. The first setting is to calculate the throughput bound with random demands, random nodes and BTs positions. The second setting is to study the impact of BT distribution patterns on the throughput bound of the network. For each setting, we make comparisons between two different node models and between OR and UR.

There are 35 nodes randomly located in an area of $1000 \mathrm{~m} \times 1000 \mathrm{~m}$. We randomly select 10 nodes as source nodes, and one node as destination node. The radio range of nodes is $150 \mathrm{~m}$. Source nodes and relay nodes send data with rate 10 packets/TS. We start with pure ad-hoc network, then add BTs randomly to the network. For the multiplechannel model, we assume that BTs have 4 wireless channels to communicate with wireless nodes. 
Figure 4 presents the throughput bound of hybrid wireless networks as the number of BTs increases. The figure shows the average values of 10 samples. From the figure, we see the growth trend of the throughput bound in random hybrid network as the number of base stations increases. When the number of base stations gets to 5 , the throughput bound of hybrid wireless network outperforms the pure ad-hoc case by more than $170 \%$ for multiplechannel model and by $125 \%$ for single-channel model (4.88 and 3.54 packets/TS compared to 2.86 packets/TS in ad-hoc case). Due to the increased capacity of BTs, the throughput bound increases higher on multiple-channel model than single-channel model. Analyzing the results in details, we found that when the number of base stations is increased, the traffic routed through the pure ad-hoc network decreases and the traffic through the infrastructure network increases. As a result, the end-to-end throughput is improved. Also for either models, OR always get significantly higher throughput than UR. The reason is that with multiple demands from multiple sources, at each hop, there are more chances for wireless nodes to forward packets through multi-nodes CRS under OR than a single relay node under UR. This makes the throughput bounds of OR much higher than UR.

For the second case study, we measure the throughput bound with the same network configuration as the first case but with three different BT's distribution patterns: random distribution, regular distribution and clustered distribution. For the regular distribution with $n$ BTs, we evenly partition the whole area into $n$ regions around the center of the area. One BT is placed at the center of each region. For the clustered distribution of BTs, we used a simple greedy scheme to add BTs one by one to the network at positions that could cover the highest number of uncovered wireless nodes. Again, we study the two models. Figure 5 shows the comparison of the throughput bounds under three BT distribution patterns with the single-channel model. With the same number of base stations deployed on the network, the throughput bound in regular distribution case is approximately as high as the throughput bound in the random distribution case, but higher than the throughput bound in the case of clustered distribution. This is because that, with the clustered BT distribution, many wireless nodes fall into the coverage of a same BT. Since each BT shares a single channel between ad-hoc transmission, the transmission through BT actually becomes the bottleneck and the bandwidth in the infrastructure network cannot be efficiently utilized. Meanwhile, for the multiple-channel model in Figure 6, the throughput bound in the case of clustered BT distribution is $15 \% \sim 20 \%$ higher than the random and regular BT distributions. In the extreme case when number of base stations is 5 , the throughput bound for 3 cases are: 4.81, 5.14 and 5.81 packets/TS respectively. The reason is: with clustered distribution, more wireless nodes can be covered with the same number of BTs, and with multiple channels, the transmissions through BTs are no longer bottleneck. This shows that the more nodes covered by BTs, the higher the throughput bound improvement. Also, in the studied case, due to random source nodes distribution, the regular BT distribution only slightly outperforms the random BT distribution.

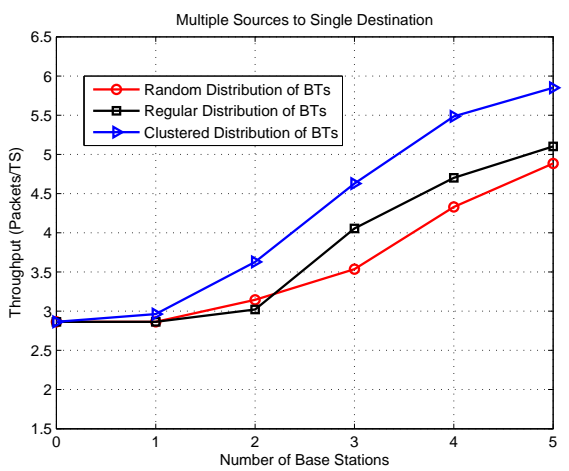

Fig. 6. Throughput bound on different distribution patterns of multi-channel BTs

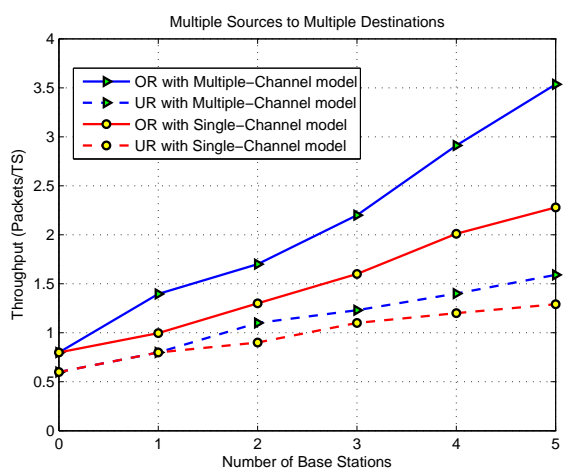

Fig. 7. Bound of throughput from Multiple Sources to Multiple Destinations

\subsection{Multiple Sources to Multiple Destinations}

For the case of Multiple Sources to Multiple Destinations, we set up a network area of $1000 \mathrm{~m}$ x 1000m. There are 10 nodes randomly placed in the area. We then configure 3 random pairs of source and destination nodes. The radio range of nodes is $150 \mathrm{~m}$. Node transmission rate is 10 packets/TS. We start with pure ad-hoc network. We then gradually add BTs one by one to random locations of the network until 5 BTs are added. The throughput 
improvement result is shown in Figure 7. From this figure, we can see that the throughput bound increases as the number of base stations increases. Throughput bound for multiple-channel model is significantly higher than the single-channel model for both OR and UR. OR always got higher throughput bound than UR. For OR, the throughput bound with 5 BTs in case of multiple-channel model is 3.5 packets/TS, which is more than four times of the throughput bound of the pure ad-hoc case ( 0.79 packets/TS) and equals to $160 \%$ throughput bound in case of single-channel model.

\section{Conclusion}

In this paper, we studied the throughput gain of OR routing schemes in hybrid wireless networks. We first extended OR to exploit the high throughput routes over infrastructure network. We then developed linear programming models to characterize the capacity of hybrid wireless networks with OR. Our models calculate the end-to-end throughput bounds from multiple source nodes to single, as well as, multiple destination nodes. Through case studies on example hybrid wireless networks, we demonstrated the throughput gain of OR in hybrid wireless networking environment. The impacts of several factors on OR performance, such as the radio range of nodes, the density and distribution pattern of BTs were evaluated in the case studies. We also demonstrated that OR got higher throughput gain than UR in both ad-hoc and hybrid wireless networks, single-channel and multiple-channel models. The current solving assumes simplified packet loss model. As a work for future direction, we will study the capacity of hybrid wireless networks with more realistic packet loss models. We used maximal CTS to calculate the throughput bounds. However it is time consuming to identify maximal CTS for large networks. We will study more efficient ways to model the conflicts between hybrid wireless links and characterize network capacity. We also plan to verify our capacity results using packet level simulations.

\section{References}

1. Kai Zeng, Wenjing Lou, and Hongqiang Zhai, "On End-to-end Throughput of Opportunistic Routing in Multirate and Multihop Wireless Networks”, IEEE Infocom 2008, Phoenix, AZ, April 15-17, 2008.

2. B. Liu, Z. Liu, and D. Towsley. "On the capacity of hybrid wireless networks". In Proc. IEEE Infocom, 2003.

3. U. Kozat and L. Tassiulas. "Throughput capacity of random ad hoc networks with infrastructure support". In Proc. of MobiCom, 2003.

4. A. Agarwal and P. Kumar, "Capacity bounds for ad hoc and hybrid wireless networks". ACM SIGCOMM Computer Communications Review, vol. 34, pp. 7181, Jul. 2004.

5. A. Zemlianov and G. de Veciana. "Capacity of ad hoc wireless networks with infrastructure support", IEEE JSAC, Volume 23, Issue 3, March 2005 Page(s): 657 - 667.

6. Qinyun Dai, Lu Rong, Honglin Hu, Capacity, "Delay and Mobility in hybrid wireless networks", IEEE International Conference on Networking, Sensing and Control, 2008. ICNSC 2008.

7. B. Liu, P. Thiran, and D. Towsley. "Capacity of a Wireless Ad Hoc Network with Infrastructure". In Proc. ACM Mobihoc, 2007.

8. S. Chachulski, M. Jennings, S. Katti, D. Katabi. "Trading structure for randomness in wireless opportunistic routing". In Proc. ACM Sigcomm, 2007.

9. H. Dubois-Ferrire, M.Grossglauser, and M. Vetterli, "Least-Cost Opportunistic Routing", 2007 Allerton Conference on Communication, Control, and Computing, Monticello IL, September 2007.

10. Douglas S. J. De Couto , Daniel Aguayo , John Bicket, Robert Morris, "A high-throughput path metric for multi-hop wireless routing", Proceedings of the 9th annual international conference on Mobile computing and networking, September 14-19, 2003, San Diego, CA, USA.

11. S. Biswas and R. Morris. "Opportunistic routing in multi-hop wireless networks". In Proceedings of the Second Workshop on Hot Topics in Networks (HotNets-II), Cambridge, MA, Nov. 2003.

12. R.C.Shah, S.Wietholter, A.Wolisz, and J.M.Rabaey, "When Does Opportunistic Routing Make Sense ?", in IEEE PerSens, Mar.2005.

13. P. Gupta and P. R. Kumar, "The capacity of wireless networks", IEEE Transactions on Information Theory, vol. 46, no. 2, Mar 2000.

14. M. Grossglauser and D. N. C. Tse, "Mobility Increases the Capacity of Ad-hoc Wireless Networks", in IEEE Infocom, 2001, pp. 1360 - 1369. 